\title{
Comparison of the CSF dynamics between patients with idiopathic normal pressure hydrocephalus and healthy volunteers
}

\author{
Johan Jacobsson, MD, ${ }^{1}$ Sara Qvarlander, PhD, ${ }^{2,3}$ Anders Eklund, PhD, ${ }^{2,3}$ and Jan Malm, MD, PhD' \\ Departments of ${ }^{1}$ Pharmacology and Clinical Neuroscience and ${ }^{2}$ Radiation Sciences-Biomedical Engineering, and ${ }^{3}$ Centre for \\ Biomedical Engineering and Physics, Umeå University, Umeå, Sweden
}

\begin{abstract}
OBJECTIVE Intracranial pressure (ICP), outflow resistance $\left(\mathrm{R}_{\text {out }}\right)$, and amplitude of cardiac-related ICP pulsations (AMPs) are established parameters to describe the CSF hydrodynamic system and are assumed, but not confirmed, to be disturbed in idiopathic normal pressure hydrocephalus (INPH). The aim of this study was to compare the CSF hydrodynamic profile between patients with INPH and healthy volunteers.
\end{abstract}

METHODS Sixty-two consecutive INPH patients (mean age 74 years) and 40 healthy volunteers (mean age 70 years) were included. Diagnosis was made by two independent neurologists who assessed patients' history, neurological status, and MRI studies. A CSF dynamic investigation through the lumbar route was performed: ICP and other CSF dynamic variables were blinded to the neurologists during the diagnostic process and were not used for establishing the diagnosis of INPH.

RESULTS $R_{\text {out }}$ was significantly higher in INPH ( $\left.R_{\text {out }} 17.1 \mathrm{vs} 11.1 ; p<0.001\right)$, though a substantial number of INPH subjects had normal $R_{\text {out }}$. There were no differences between INPH patients and controls regarding ICP (mean $11.5 \mathrm{~mm} \mathrm{Hg}$ ). At resting pressure, there was a trend that AMP in INPH was increased ( 2.4 vs $2.0 \mathrm{~mm} \mathrm{Hg} ; p=0.109)$. The relationship between AMP and ICP was that they shared the same slope, but the curve was significantly shifted to the left for INPH (reduced $\mathrm{P}_{0}[\mathrm{p}<0.05]$; i.e., higher AMP for the same ICP).

CONCLUSIONS This study established that the CSF dynamic profile of INPH deviates from that of healthy volunteers and that INPH should thus be regarded as a disease in which intracranial hydrodynamics are part of the pathophysiology.

Clinical trial registration no.: NCT01188382 (clinicaltrials.gov)

https://thejns.org/doi/abs/10.3171/2018.5.JNS173170

KEYWORDS normal pressure hydrocephalus; gait disorders; cerebrospinal fluid; dementia; intracranial pressure; pulsatility

$\mathrm{N}$ ORMAL pressure hydrocephalus (NPH) is a neurological condition for which a disturbance in the CSF dynamic system has been postulated. The rationale comes from the dilated ventricles and that CSF shunting is an effective treatment. However, there is no consensus on the specifics of the CSF dynamic disturbance, which makes both the diagnosis and selection of patients for shunt surgery challenging. ${ }^{21}$

In recent years, the idiopathic form of the condition (idiopathic NPH [INPH]) has been the focus for research, and standardized guidelines for INPH were established in $2005 .{ }^{16}$ Despite these guidelines, diagnosis is not straightforward. For instance, there is a high degree of comor- bidity in INPH, ${ }^{12}$ and there are many disorders that may mimic the INPH symptoms. The INPH guidelines depend on clinical features, radiology with communicating hydrocephalus, and a normal intracranial pressure (ICP). ${ }^{19}$ The guidelines also contain objective technical and laboratory tests supporting the diagnosis and supplementary tests for selecting patients for surgery. ${ }^{15}$ The recommended tests all have grounds in CSF dynamics.

CSF dynamics are easily assessed with a lumbar infusion investigation and can be described in standardized terms based on pressure, flow, and volume. ${ }^{5}$ CSF outflow resistance $\left(R_{\text {out }}\right)$ is defined as the relationship between pressure and flow. The corresponding pressure-versus-

ABBREVIATIONS AMP = amplitude of cardiac-related ICP pulsation; $\mathrm{C}_{\text {out }}=$ conductance to $C S F$ outflow; ICP = intracranial pressure; INPH = idiopathic normal pressure hydrocephalus; MMSE = Mini-Mental State Examination; NPH = normal pressure hydrocephalus; $P_{d}=$ dural venous pressure; $P_{0}=$ (theoretical) pressure at infinite compliance; $R_{\text {out }}=$ outflow resistance; $R P P C=$ relative pulse pressure coefficient.

SUBMITTED December 18, 2017. ACCEPTED May 29, 2018.

INCLUDE WHEN CITING Published online November 16, 2018; DOI: 10.3171/2018.5.JNS173170. 


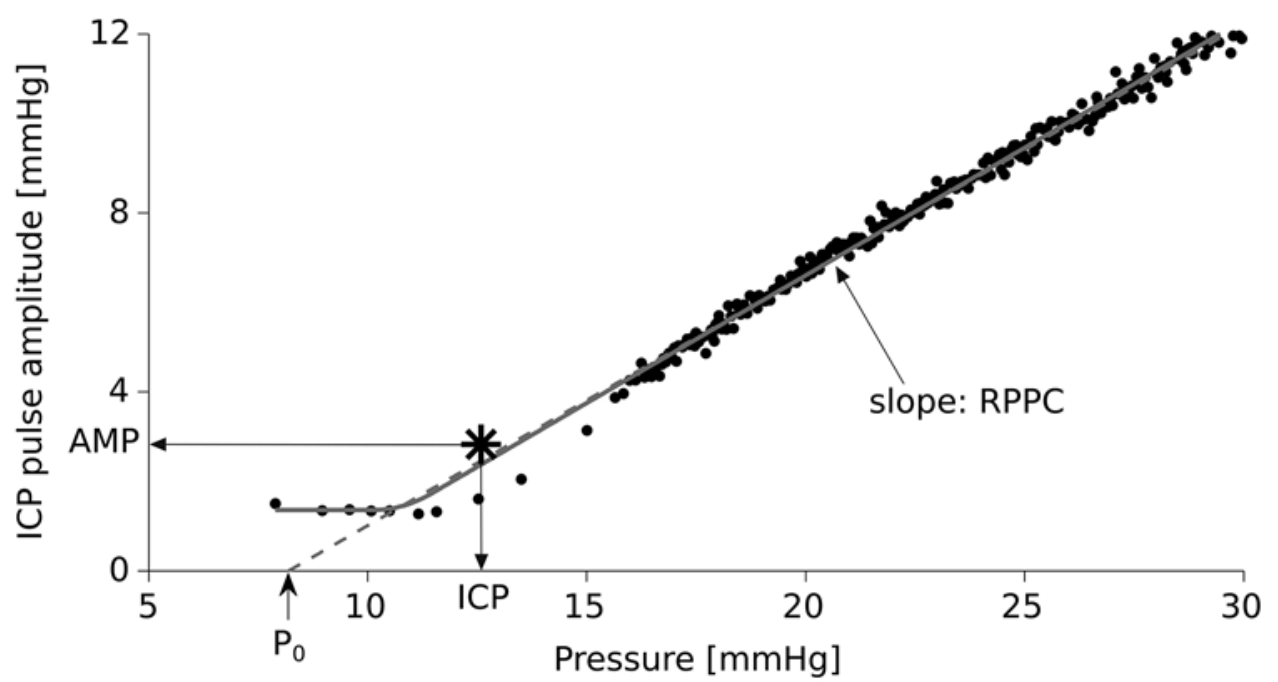

FIG. 1. Pressure and pulse amplitude data from a single INPH patient, illustrating the calculations of RPPC and $P_{0}$ and showing the resting ICP and AMP values.

volume relationship can be illustrated by the cardiac-related ICP pulsations at different pressure levels; typically the pulse pressure amplitude increases as the pressure is raised (Fig. 1). ${ }^{3}$

A CSF dynamic investigation is invasive and requires lumbar puncture; thus, very few healthy individuals have been investigated with this method..$^{13}$ The presumed typical CSF dynamic profile of INPH (i.e., elevated $\mathrm{R}_{\text {out }}$, ICP, and pulse amplitudes) has therefore never been validated against age-matched healthy subjects. ${ }^{11}$

The aim of this study was to determine if the CSF hydrodynamic profile of INPH is disturbed in relation to that seen in healthy elderly volunteers.

\section{Methods}

\section{Healthy Subjects}

The material on the healthy subjects has been previous-

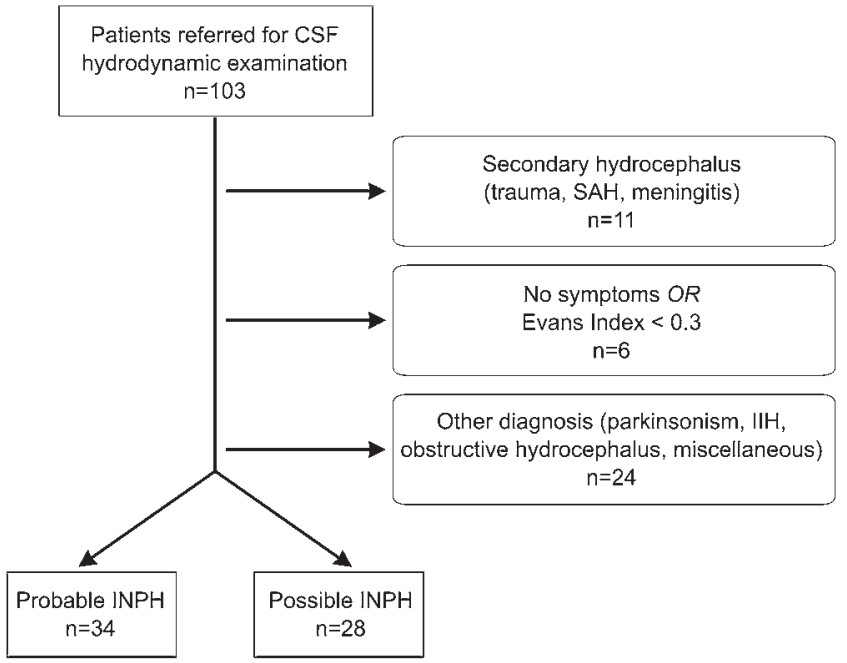

FIG. 2. Flowchart illustrating inclusions and exclusions of patients. IIH = idiopathic intracranial hypertension; $\mathrm{SAH}=$ subarachnoid hemorrhage. ly published. ${ }^{13}$ In summary, 40 healthy individuals aged 61-81 years were examined. Volunteers had no diabetes, no previous disorder of the CNS, not more than two vascular risk factors, no medication directly influencing the nervous system, no heart disease, and no contraindications to MRI or lumbar puncture. The score on the Mini-Mental State Examination (MMSE) had to exceed 28 points.

\section{INPH Patients}

The case records of 103 consecutive patients (aged > 40 years) with ventriculomegaly, referred to our department from 2007 to 2010 because of suspicion of INPH, were reviewed. Patients had undergone MRI and a CSF dynamic investigation. A flowchart illustrating inclusions and exclusions is shown in Fig. 2. After classification (as below), 34 probable and 28 possible INPH cases were included. Clinical features of INPH patients and healthy controls are summarized in Table 1. Forty-one cases were excluded. Reasons for exclusion were as follows: secondary hydrocephalus (subarachnoid hemorrhage, meningitis, trauma; $\mathrm{n}=11$ ), other diagnosis (obstructive hydrocephalus, idiopathic intracranial hypertension, parkinsonism; $\mathrm{n}$ $=24)$, and Evans index $<0.3$ or lack of symptoms $(n=6)$.

Case records and radiological images of each patient were reviewed as follows: two senior neurologists had access to a de-identified copy of each initial outpatient record. The material available included clinical history, neurological/physical examination status, MMSE score, and a written report of brain MRI findings (CT scans for two patients), including a measure of the ventricular size (Evans index). It is important to stress that the neurologists were blinded to the results from the CSF hydrodynamic investigation such as $\mathrm{R}_{\text {out }}$ and compliance. CSF opening pressure (i.e., ICP) was also kept blinded to the assessors. They also did not have access to laboratory findings, final diagnosis, or the decision on shunt placement. The examiners worked independently of each other. Disagreement between reviewers (39 cases) was typically either on the degree of comorbidity or the features or severity of the gait 
TABLE 1. Baseline characteristics of patients and healthy controls

\begin{tabular}{lcccc}
\hline Characteristic & Probable INPH $(n=34)$ & Possible INPH $(n=28)$ & All INPH $(n=62)$ & Healthy Controls $(n=40)$ \\
\hline Age, yrs & $75(6)$ & $74(7)$ & $74(6)$ & $70(6)$ \\
\hline Sex, M/F ratio & $22 / 12$ & $21 / 7$ & $43 / 19$ & $18 / 22$ \\
\hline MMSE score & $24(4)$ & $25(5)$ & $25(4)$ & $29(1)$ \\
\hline Evans index & $0.39(0.04)$ & $0.38(0.03)$ & $0.39(0.04)$ & $0.28(0.03)$ \\
\hline
\end{tabular}

All data shown are presented as the mean (SD), except sex.

disorders. By-default cases in which the discrepancy was between probable and possible INPH (19 cases) were classified as "possible." Cases excluded by one reviewer but included by the other (20 cases) were subject to consensus discussion. In the end, 17 of these cases were excluded.

The diagnosis of INPH was based on the INPH guidelines. ${ }^{19}$ Cases were classified as "probable" INPH irrespective of the CSF opening pressure (as this was not known by the assessors). Communicating hydrocephalus was defined as ventricular enlargement (Evans ratio $>0.3$ ) without obstruction to CSF flow. Supportive features such as callosal angle or aqueductal flow were not scored. The guidelines state that 2 of 9 typical gait features (such as decreased step height, step length, or velocity) should be present. However, the gait disturbance was often described as "typical" in the clinical history, including various descriptions of its characteristics. Cognitive decline was defined as either an MMSE score $<25$ or a combination of an MMSE score of $<30$ and a cognitive decline reported by the patient or relatives.

Patients in whom INPH was defined as "possible" had only 1 of the 3 INPH cardinal symptoms, or they had extensive comorbidity, but, in our final judgment, the symptoms were not entirely attributable to that comorbidity, as stated in the guidelines. Controls and patients were not matched, so there was an abundance of males among the patients. Patients were slightly older than controls, ranging from 59 to 85 years at the time of their investigation (Table 1).

\section{CSF Dynamics}

Patients and controls were investigated using a CSF dynamic apparatus (Celda, Likvor AB) with an automated infusion protocol. The apparatus is approved within the European Community and entails lumbar puncture using two needles, one for recording the pressure and the other for manipulating the pressure by infusion of artificial CSF. In the measurement procedure, patients lie relaxed on their backs with a clear reference level for the pressure measurement (at the external auditory canal). Pressure was sampled and recorded at $100 \mathrm{~Hz}$; the data were postprocessed and all study parameters calculated using MATLAB (The MathWorks Inc.). Resting ICP was recorded in the supine position for 15-20 minutes, and the mean value of the last 5 minutes was defined as the ICP. The baseline amplitude of the cardiac-related ICP pulsations (AMPs) was determined from the same period using an algorithm developed in-house. ${ }^{7}$ For the majority of the subjects, a constant-pressure infusion protocol was used, where the pressure was elevated in steps and regulated to remain at 4-6 predetermined levels. ${ }^{1}$ The conductance to
CSF outflow $\left(\mathrm{C}_{\text {out }}\right.$; i.e., the inverse of $\left.\mathrm{R}_{\text {out }}\right)$ was calculated as the slope of a linear regression of the flow rates required to maintain the pressure levels versus the achieved mean pressures at each level. This method is thus independent of the baseline ICP. $\mathrm{R}_{\text {out }}$ was calculated with a method that takes baseline ICP into account, by averaging the achieved pressure levels and dividing the difference between this mean pressure and the baseline ICP, with the average required flow rate. In case of problems using the constantpressure method, a constant (flow) infusion method was used. ${ }^{20}$ In such cases, $R_{\text {out }}$ was determined using the same calculation as above, with the average pressure at the plateau as the mean pressure, and the infusion flow rate (1.5 $\mathrm{ml} /$ minute) as the average flow. $\mathrm{C}_{\text {out }}$ was calculated as the inverse of $R_{\text {out }}$ in these cases.

The relative pulse pressure coefficient (RPPC) $)^{10}$ was estimated using linear regression of pairs of pressure and pulse amplitude values (averaged over 10 cardiac cycles) measured during the infusion (at each constant-pressure level or during the entire constant [flow] infusion). The slope of the linear regression line is an estimate of RPPC, and $\mathrm{P}_{0}$ is where this line intersects the pressure axis (i.e., the pressure theoretically corresponding to 0 pulse amplitude) (Fig. 1), RPPC relates to the compliance of the CSF system (specifically to how compliance changes with ICP), and $\mathrm{P}_{0}$ is a theoretical reference pressure, corresponding to the pressure at infinite compliance, possibly relating to pressure in the venous sinuses. ${ }^{2}$ These parameters were defined according to equations designed by Avezaat and van Eijndhoven, ${ }^{2}$ based on the Marmarou model. ${ }^{17}$

\section{Statistical Analysis}

One-way ANOVA with post hoc Bonferroni tests was used for comparing all 3 groups (probable INPH, possible INPH, and controls). Comparisons between all patients and controls were performed using two-sample t-tests. Significance was set to $\mathrm{p}<0.05$.

\section{Ethical Approval}

The study regarding healthy subjects was approved by the local ethics committee, and an informed consent form was signed by each participant before inclusion (clinicaltrials.gov no. NCT01188382). After separate approval from the local ethics committee, data regarding the hydrocephalus patients were de-identified and analyzed.

\section{Results}

The results are shown in Table 2. There was no difference in ICP among the 3 groups (controls, possible INPH, 
TABLE 2. CSF dynamic parameters for the INPH patients and healthy controls

\begin{tabular}{lcccc}
\hline \multicolumn{1}{c}{ Parameter } & Probable INPH $(n=34)$ & Possible INPH $(n=28)$ & All INPH $(n=62)$ & Healthy Controls $(n=40)$ \\
\hline $\mathrm{ICP}, \mathrm{mm} \mathrm{Hg}$ & $11.3(2.2)$ & $11.8(3.0)$ & $11.5(2.6)$ & $11.5(2.3)$ \\
\hline $\mathrm{R}_{\text {out }}, \mathrm{mm} \mathrm{Hg} /(\mathrm{ml} / \mathrm{min})$ & $17.93(7.36)^{*}$ & $16.18(9.38)^{*}$ & $17.14(8.3)^{*}$ & $11.06(7.52)$ \\
\hline $\mathrm{C}_{\text {out }}, \mathrm{\mu l} / \mathrm{sec} / \mathrm{kPa}$ & $6.38(3.65)^{*}$ & $7.37(5.73)^{*}$ & $6.83(4.7)^{*}$ & $14.56(11.84)$ \\
\hline $\mathrm{AMP}, \mathrm{mm} \mathrm{Hg} \dagger$ & $2.4(1.3)$ & $2.4(1.3)$ & $2.4(1.3)$ & $2.0(1.3)$ \\
\hline $\mathrm{RPPC} \dagger$ & $0.59(0.22)$ & $0.61(0.23)$ & $0.60(0.23)$ & $0.62(0.23)$ \\
\hline $\mathrm{P}_{0}, \mathrm{~mm} \mathrm{Hgt}$ & $6.9(3.4)^{*}$ & $8.4(3.1)^{*}$ & $7.6(3.3)^{*}$ & $9.1(2.7)$ \\
\hline
\end{tabular}

All data are presented as the mean (SD).

* Indicates statistically significant differences compared to controls $(p<0.05)$; no statistically significant differences were found between patients with probable and possible INPH.

† Two healthy control subjects were excluded from analysis of ICP pulsatility due to signal issues $(n=38)$.

and probable INPH; ANOVA, $p=0.717$ ). $\mathrm{R}_{\text {out }}$ was higher in both probable and possible INPH cases versus healthy controls (Fig. 3; ANOVA, $p=0.001$ ), while the difference between probable and possible INPH was not significant $(\mathrm{p}=1.000)$. Baseline pulse amplitudes did not differ significantly between any of the groups (ANOVA, $p=0.279$ ), but there was a tendency toward higher amplitudes among patients rather than controls (mean difference $0.4 \mathrm{~mm} \mathrm{Hg}$, $\mathrm{p}=0.109$ ). RPPC was similar in all groups (ANOVA, $\mathrm{p}=$ 0.797), illustrating that the slope between AMP and ICP was the same for all groups. However, the $\mathrm{P}_{0}$ was significantly lower among patients than among controls ( $p$ $=0.020$ ), indicating that the curve was shifted to the left for INPH, with no significant difference between probable and possible INPH cases. There was overlap of the distributions among all groups for all parameters, including $\mathrm{R}_{\text {out }}, \mathrm{C}_{\text {out }}$, and $\mathrm{P}_{0}$, as illustrated in the box plots in Fig. 3.

\section{Discussion}

This study provides evidence for differences in CSF dynamics between INPH patients and healthy elderly controls. Normal ICP and high $\mathrm{R}_{\text {out }}$ characterize the CSF dynamic profile of patients with INPH. Furthermore, the pulsatility curve (Fig. 1), which relates compliance to ICP, was shifted to the left for the INPH patients, corresponding to the trend toward increased ICP pulse amplitude at baseline (Fig. 3). Our study is important for the patient groups, as its findings can help to improve diagnostics, increase knowledge of INPH pathophysiology, and advance our understanding of the changes in CSF dynamics caused by a CSF shunt, which in turn can render insight into how shunting can lead to clinical improvement.

\section{Intracranial Pressure}

The selection of INPH patients in this study closely adhered to the INPH guidelines, but not including the criteria for ICP. The mean ICP of the INPH patients was the same as it was for healthy controls, and none of the INPH patients had elevated ICP. Although the name "normal" pressure hydrocephalus suggests a normal ICP, it has been speculated that INPH may be associated with a slight increase in ICP. ${ }^{14}$ The study suggesting this was performed before the publication of the INPH guidelines, and the patient group differed from the present definition of INPH.
The diagnostic criteria of the guidelines are likely good at excluding secondary causes of NPH, where findings of increased ICP are more common. In the present study, the use of the guidelines provided an INPH population in which ICP was clearly normal, which strongly implies that the upper ICP threshold of the guidelines should be adjusted downward. We suggest that an ICP $<15 \mathrm{~mm} \mathrm{Hg}$ should be used for the diagnosis of probable INPH in a future revision of the guidelines.

\section{CSF $R_{\text {out }}$}

At the group level, there was a clear difference in $\mathrm{R}_{\text {out }}$, with the INPH group having higher values. This is in agreement with a previous study in which INPH was compared to historical controls..$^{14}$ However, in the present study there was overlap between the two populations, and $65 \%$ of the INPH subjects fell into the normal range of $R_{\text {out }}$ for the healthy elderly cohort. ${ }^{13}$ Thus, $R_{\text {out }}$ is not sufficient as a diagnostic parameter, but it is important to note that while a low/normal $R_{\text {out }}$ does not exclude INPH, a high $R_{\text {out }}$ is strongly supportive of an INPH diagnosis. We suggest $\mathrm{R}_{\text {out }}$ could be used as a supplemental parameter in the diagnostic procedure, when, for example, monosymptomatic patients with a high $\mathrm{R}_{\text {out }}$ could be classified as probable cases of INPH.

\section{ICP Pulsatility}

The significantly lower $\mathrm{P}_{0}$ values of the INPH patients revealed in this study (Fig. 3D) suggest that the pulsatility curve (Fig. 1) was shifted toward the left in INPH. Given that both ICP and RPPC (the slope of the curve) were normal in patients with INPH, this leftward shift could explain the tendency toward increased ICP pulse amplitudes at baseline, as the same ICP value corresponds to a higher pulse amplitude value if the curve is shifted to the left. The ICP pulse amplitudes have previously been suggested to be higher in NPH. ${ }^{8,9}$ Those studies were hampered by having too few subjects and/or a mix of idiopathic and secondary NPH. ${ }^{8}$ Several of the suggested hypotheses on INPH pathophysiology include increased ICP pulsatility as a relevant aspect, but based on our results, it may not be sufficient to explain all cases of INPH. Similarly to $\mathrm{R}_{\text {out }}$, we found a large overlap between the groups (Fig. 3), indicating that the disturbance of CSF dynamics is more complex. 


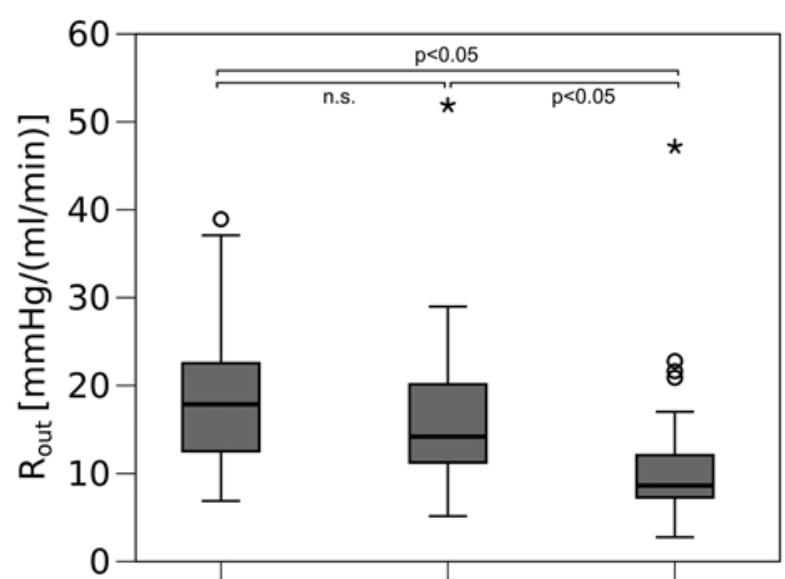

A probable INPH possible INPH healthy controls

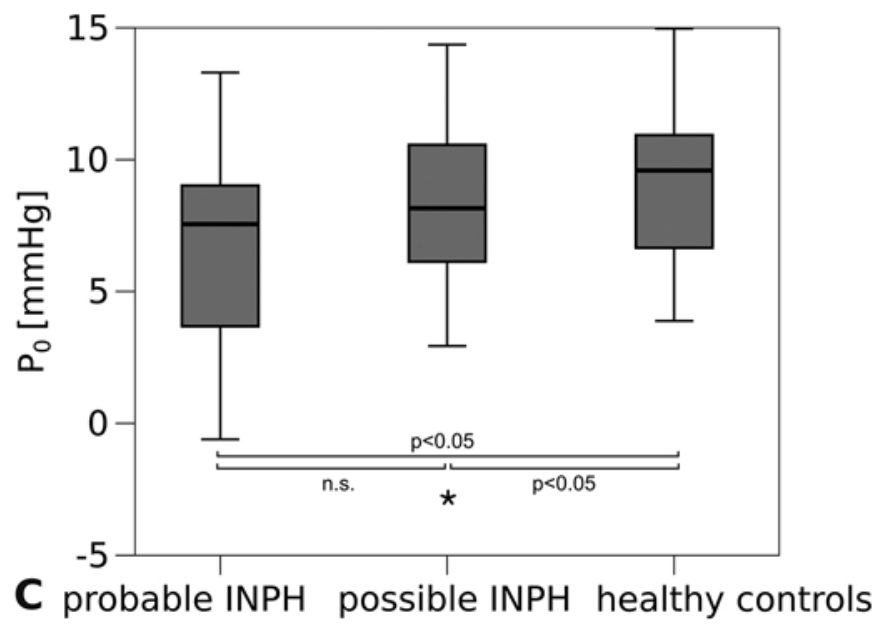

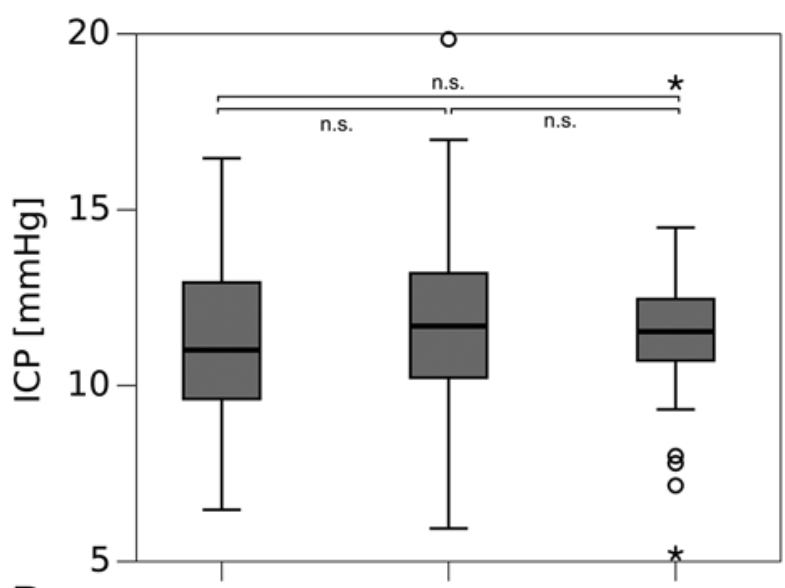

B probable INPH possible INPH healthy controls

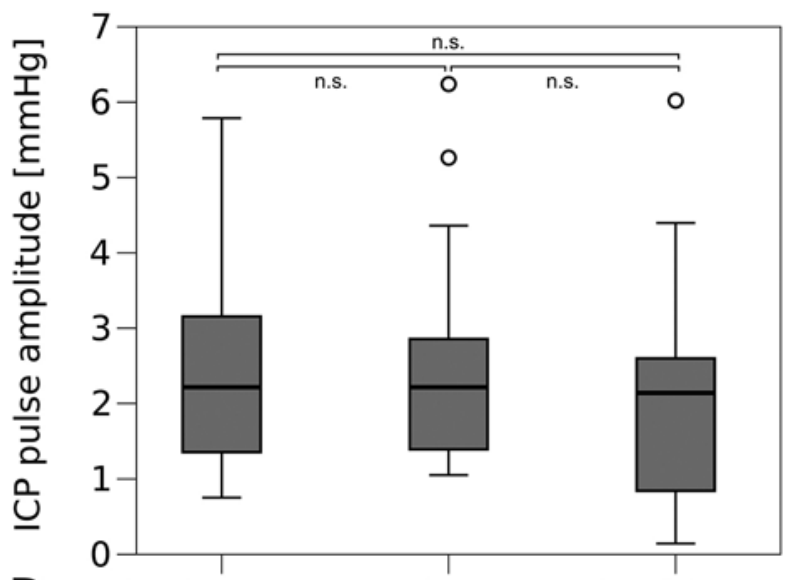

probable INPH possible INPH healthy controls

FIG. 3. Box plots illustrating the differences in $R_{\text {out }}(A), I C P(B), P_{0}(C)$, and $A M P(D)$ among probable-INPH patients, possibleINPH patients, and controls. Two healthy controls were excluded from analysis of ICP pulsatility (including RPPC and $\mathrm{P}_{0}$ ) due to signal issues; thus, 38 healthy subjects are included in panels $C$ and D. The line, box, and whiskers represent the median, interquartile range, and range, respectively, while circles and asterisks represent statistical outliers; brackets illustrate statistical comparisons ( $p<0.05$ or n.s. [not significant]).

\section{Pulsatility and Prediction for Shunt Surgery}

$\mathrm{P}_{0}$ and the pulsatility curve can facilitate an understanding of how $\mathrm{R}_{\text {out }}$ and ICP pulse amplitude are linked. $\mathrm{P}_{0}$ was introduced to improve the fit of the mathematical model to clinical data, and it has been proposed as relating to dural venous pressure $\left(\mathrm{P}_{\mathrm{d}}\right){ }^{2}$ ICP also relates to $\mathrm{P}_{\mathrm{d}}$ according to the Davson equation, ${ }^{6}$ and a reduction of $\mathrm{P}_{0}$ should therefore be accompanied by reduction of the ICP, whereas an increased $R_{\text {out }}$ would suggest an increased ICP. Baseline ICP in this study was normal in INPH patients, indicating that the elevated $\mathrm{R}_{\text {out }}$ and reduced $\mathrm{P}_{0}$ (and thus potentially reduced $\mathrm{P}_{\mathrm{d}}$ ), on the group level, balance one another, though with the result that INPH has slightly increased ICP pulse amplitude. This compensating CSF dynamic profile of INPH may thus help to explain why the parameters $R_{\text {out }}$, $\mathrm{P}_{0}$, and ICP pulse amplitude can correspond to normal ICP but still describe a pattern of a disturbed CSF dynamics. The physiological interaction between resistance in the outflow pathways, the compliance of the craniospinal compartment, venous pressure, and the ICP pulse generated by cardiac-related arterial volume variations also indicates that isolated analysis of single variables are not sufficient to characterize a patient's CSF dynamics. Future research may thus benefit from taking into account several parameters for both outcome prediction and diagnostics in INPH. Patients with a low $\mathrm{R}_{\text {out }}$ may, for example, still present with abnormal pulsatility-related dynamics, which could indicate a positive outcome from shunt surgery..$^{18}$ We believe that a more in-depth analysis of the pulsatility curve may yield a more comprehensive picture of the compliance-related CSF dynamics than, for example, AMP, and a multicenter study investigating this approach has been initiated at hospitals in Sweden and Finland.

\section{Limitations}

Measurements of ICP and CSF dynamics always entail a certain measurement-related uncertainty; however, the apparatus for CSF dynamic evaluation that was used in this study is commercially available and standardized and has high measurement accuracy. ${ }^{1}$ In our center, we have used 
the method for many years prior to the study period, and we believe we have achieved reliable estimates. The ICP pulse amplitude is dampened in its transfer from cranial to lumbar regions and thus is reduced in magnitude; ${ }^{4}$ however, the effect should be similar for INPH and controls.

The control individuals were collected as a reference material for CSF dynamics in healthy elderly people, ${ }^{13}$ in large part prior to the investigations of the INPH patients presented in this study. Thus, patients and controls were not directly matched for sex and age. This presents a limitation to the study; however, a post hoc analysis with corrections for age and sex (multiple linear regression analysis) suggested that this did not substantially affect the results and did not change the conclusions of this study. A restriction of the study was that, since the investigated CSF dynamic parameters were subsequently used in the clinical selection of patients for shunt surgery, results of an analysis regarding treatment and outcome would have been biased and could not be properly performed.

\section{Conclusions}

We found that the CSF dynamic profile of INPH patients was characterized by an increased $\mathrm{R}_{\text {out }}$, normal ICP, a tendency toward increased ICP pulse amplitude, and a leftward shift of the pulsatility curve, that is, a reduced $\mathrm{P}_{0}$. This established that the CSF dynamic profile of INPH deviates from that of healthy individuals and that INPH thus should be regarded as a disease in which intracranial hydrodynamics are a part of the pathophysiological description. For further work, the findings in this paper suggest a comprehensive approach to CSF dynamics analysis that, in addition to $\mathrm{R}_{\text {out }}$, includes the pulsatility curve with assessment of $\mathrm{P}_{0}$.

\section{Acknowledgments}

The study received funding from the Swedish Research Council grant no. 2015-05616 (awarded to Prof. Eklund).

\section{References}

1. Andersson N, Malm J, Bäcklund T, Eklund A: Assessment of cerebrospinal fluid outflow conductance using constantpressure infusion-a method with real time estimation of reliability. Physiol Meas 26:1137-1148, 2005

2. Avezaat CJ, van Eijndhoven JH: Clinical observations on the relationship between cerebrospinal fluid pulse pressure and intracranial pressure. Acta Neurochir (Wien) 79:13-29, 1986

3. Avezaat CJ, van Eijndhoven JH, Wyper DJ: Cerebrospinal fluid pulse pressure and intracranial volume-pressure relationships. J Neurol Neurosurg Psychiatry 42:687-700, 1979

4. Behrens A, Lenfeldt N, Ambarki K, Malm J, Eklund A, Koskinen LO: Intracranial pressure and pulsatility index. Neurosurgery 69:E1033-E1034, 2011

5. Czosnyka M, Czosnyka Z, Momjian S, Pickard JD: Cerebrospinal fluid dynamics. Physiol Meas 25:R51-R76, 2004

6. Davson H, Domer FR, Hollingsworth JR: The mechanism of drainage of the cerebrospinal fluid. Brain 96:329-336, 1973

7. Farahmand D, Qvarlander S, Malm J, Wikkelsö C, Eklund A, Tisell M: Intracranial pressure in hydrocephalus: impact of shunt adjustments and body positions. J Neurol Neurosurg Psychiatry 86:222-228, 2015
8. Foltz EL, Aine C: Diagnosis of hydrocephalus by CSF pulsewave analysis: a clinical study. Surg Neurol 15:283-293, 1981

9. Greitz D: Radiological assessment of hydrocephalus: new theories and implications for therapy. Neurosurg Rev 27:145-167, 2004

10. Lenfeldt N, Andersson N, Agren-Wilsson A, Bergenheim AT, Koskinen LOD, Eklund A, et al: Cerebrospinal fluid pulse pressure method: a possible substitute for the examination of B waves. J Neurosurg 101:944-950, 2004

11. Malm J, Eklund A: Idiopathic normal pressure hydrocephalus. Pract Neurol 6:14-27, 2006

12. Malm J, Graff-Radford NR, Ishikawa M, Kristensen B, Leinonen V, Mori E, et al: Influence of comorbidities in idiopathic normal pressure hydrocephalus - research and clinical care. A report of the ISHCSF task force on comorbidities in INPH. Fluids Barriers CNS 10:22, 2013

13. Malm J, Jacobsson J, Birgander R, Eklund A: Reference values for CSF outflow resistance and intracranial pressure in healthy elderly. Neurology 76:903-909, 2011

14. Malm J, Kristensen B, Karlsson T, Fagerlund M, Elfverson J, Ekstedt J: The predictive value of cerebrospinal fluid dynamic tests in patients with the idiopathic adult hydrocephalus syndrome. Arch Neurol 52:783-789, 1995

15. Marmarou A, Bergsneider M, Klinge P, Relkin N, Black PM: The value of supplemental prognostic tests for the preoperative assessment of idiopathic normal-pressure hydrocephalus. Neurosurgery 57 (3 Suppl):S17-S28, ii-v, 2005

16. Marmarou A, Bergsneider M, Relkin N, Klinge P, Black PM: Development of guidelines for idiopathic normal-pressure hydrocephalus: introduction. Neurosurgery 57 (3 Suppl):S1S3, ii-v, 2005

17. Marmarou A, Shulman K, LaMorgese J: Compartmental analysis of compliance and outflow resistance of the cerebrospinal fluid system. J Neurosurg 43:523-534, 1975

18. Qvarlander S, Lundkvist B, Koskinen LOD, Malm J, Eklund A: Pulsatility in CSF dynamics: pathophysiology of idiopathic normal pressure hydrocephalus. J Neurol Neurosurg Psychiatry 84:735-741, 2013

19. Relkin N, Marmarou A, Klinge P, Bergsneider M, Black PM: Diagnosing idiopathic normal-pressure hydrocephalus. Neurosurgery 57 (3 Suppl):S4-S16, ii-v, 2005

20. Sundström N, Andersson K, Marmarou A, Malm J, Eklund A: Comparison between 3 infusion methods to measure cerebrospinal fluid outflow conductance. J Neurosurg 113:12941303,2010

21. Williams MA, Malm J: Diagnosis and treatment of idiopathic normal pressure hydrocephalus. Continuum (Minneap Minn) 22 (2 Dementia):579-599, 2016

\section{Disclosures}

A. E. and J. M. have received revenues from Likvor AB for a patent.

\section{Author Contributions}

Conception and design: Jacobsson, Eklund, Malm. Acquisition of data: Jacobsson, Malm. Analysis and interpretation of data: all authors. Drafting the article: Jacobsson. Critically revising the article: all authors. Reviewed submitted version of manuscript: all authors. Approved the final version of the manuscript on behalf of all authors: Jacobsson. Statistical analysis: Qvarlander, Eklund. Study supervision: Malm.

\section{Correspondence}

Johan Jacobsson: Umeå University, Umeå, Sweden. johan. jacobsson@umu.se. 